\title{
Development of a Mixed Antigen Agar Gel Enzyme Assay (AGEA) for the Detection of Antibodies to Poxvirus in Chicken and Turkey Sera
}

\author{
Theodros TADESE ${ }^{1)}$, E.A. POTTER ${ }^{1)}$ and W.M. REED ${ }^{1)}$ \\ 1) Avian Disease Research Laboratory, Department of Pathobiology and Diagnostic Investigation and Animal Health, Diagnostic \\ Laboratory, A510 East Fee Hall, Michigan State University, East Lansing, MI 48824, U.S.A.
}

(Received 20 February 2002/Accepted 17 September 2002) ABSTRACT. A mixed-antigen agar gel enzyme assay (AGEA) was developed to detect antibodies to poxviruses in chicken and turkey sera.
The assay combines the principles of immunodiffusion and enzyme assay. For the detection of antibodies to fowl poxvirus (FP), pigeon
poxvirus (PP) and turkey poxvirus (TP) in turkey serum samples, the three antigens were combined to form a mixed-antigen assay. To
screen for antibodies to FP and PP in chicken serum samples, the two antigens were combined. When FP and PP viruses were combined
as antigens, the sensitivity for chicken sera was $64 \%$ but the sensitivity of the agar gel precipitation test (AGPT) was $34 \%$ (P $<0.001)$.
When antibodies were detected in turkey sera using the mixed antigens, the AGEA had a sensitivity of $66.4 \%$ while that of AGPT was
$25 \%(\mathrm{P}<0.001)$.

KEY WORDS: AGEA, avian, poxvirus.

J. Vet. Med. Sci. 65(2): 255-258, 2003

Fowl pox is one of the oldest diseases of chickens and turkeys. The etiological agent of the disease is a large DNA virus belonging to the genus Avipoxvirus of the family Poxviridae. The disease is characterized by the development of discrete, nodular, proliferative skin lesions on the unfeathered parts of the body (cutaneous form) or fibrino-necrotic and proliferative lesions in the mucous membrane of the upper respiratory tract, mouth and esophagus (diphtheritic form) [19]. The diagnosis of fowl pox has been based on clinical and/or histopathological observations followed by isolation of poxvirus in embryonated chicken eggs for virus identification. Although egg inoculation is simple, the method of diagnosis of pox is time consuming and requires a regular supply of 10-12 day-old susceptible embryonated eggs. Development of a rapid diagnostic technique for screening pox in flocks is thus needed. Serological tests such as, agar gel precipitation test (AGPT) [1, 8, 15, 19-21], passive hemagglutination test (PHT) [8, 18], neutralization test (NT) [4], and compliment fixation test (CFT) [19], have been used for detection of antibodies to fowl poxvirus. The AGPT has limitations because it only detects precipitating antibodies for a short period of time after infection. Both the PHT and CFT have not found any favorable use, although the PHT detects antibodies earlier than the AGPT. The NT is not practical for regular use as a diagnostic test. The enzyme-linked immunosorbent assay (ELISA) is a rapid, sensitive, and reproducible serological test $[4,12]$, but has low post-vaccinal and post-challenge serological reactions [12]. AGEA has been used to detect and quantitate antibodies to different antigens $[5,7,9-11,17]$. The AGEA combines the principles of radial immunodiffusion and ELISA and has been found to be a simple and reliable serological test which requires no specialized facilities or equipment.

In the present study, the development of a mixed antigen agar gel enzyme assay for the detection of antibodies to poxviruses is described. Mixed antigens comprising either fowl poxvirus (FP) and pigeon poxvirus (PP) antigens or fowl poxvirus (FP), pigeon poxvirus (PP) and turkey poxvirus (TP) antigens were used. The sensitivity and specificity of the AGEA were compared to the AGPT.

Chicken embryo fibroblast (CEF) cultures were prepared from 11-day-old embryos of RPRL line 0 chickens, as previously described [16]. Three poxviruses, FP, PP and TP vaccine viruses were propagated in primary CEF cultures. The three viruses propagated in CEF cultures were purified by sodium diatrizoate density gradient centrifugation [14]. Antibodies to FP, PP and TP were produced as previously described [14]. Briefly FP, PP, or TP infected CEF cultures were scraped off plates and sonicated for four cycles; 15 seconds each with 45 seconds of cooling on ice between cycles. A Freund's complete adjuvant was homogenized with an equal volume of the purified virus material. One $\mathrm{ml}$ of the homogenate was inoculated intramuscularly into two, 12-week-old specified-pathogen-free (SPF) chicken. The dose per chicken contained the equivalent of approximately $2 \times 10^{7} \mathrm{FP}, \mathrm{PP}$, or TP infected cells. Following 2 weeks primary inoculation, a second inoculation was given to each chicken. Finally, antigen comprising equal volumes of purified virus material homogenized with Freund's incomplete adjuvant was administered 2 weeks after the second inoculation. Blood samples were collected from inoculated chickens two weeks after the last inoculation and sera were obtained. While antisera produced as described above served for test standardization, sera were also obtained from 10 sham-inoculated chickens and 10 sham-inoculated (adjuvant in phosphate-buffered saline (PBS)) turkeys. Sera from chickens and turkeys inoculated with chicken embryonated fibroblast cells were also included as serum controls. Known positive and negative control sera were from SPAFAS Ltd. (CT). Sera (324 samples) were obtained from commercial turkey breeders vaccinated at 3 weeks of age with FP, PP and TP vaccines according to recommendations 
of the vaccine manufacturer. Chicken sera (150 samples) samples were from birds experimentally vaccinated at 3 weeks of age with both FP and PP vaccines. All showed 'takes' 7 days post vaccination and blood was drawn two weeks post vaccination. To detect antibodies specific for the poxviruses used in turkeys, purified FP, PP and TP antigens were used as a trivalent-antigen in the AGPT and AGEA. For detecting antibodies in chicken sera, a bivalentantigen comprising FP and PP antigens were used. The antigen concentrations were determined by a spectrophotometer (Spectronic 501/601) at $280 \mathrm{~nm}$. Each purified antigen, as described above, was reconstituted at six different concentrations of $0.1,0.5,1.0,1.5,2.0$ and $2.5 \mathrm{mg} / \mathrm{m} l$ in carbonatebicarbonate coating buffer. Coating of antigen was performed as described [4]. In brief, polystyrene petri dishes or plates $(60 \mathrm{~mm}$ in diameter) were coated with the mixed antigen $(2 \mathrm{~m} l)$. The optimal coating concentration for each antigen in AGEA was determined by checkerboard titrations as described $[2,3]$. In these titrations, dilutions of $1: 100$, $1: 500,1: 1,000,1: 1,500$ and 1:2,000 of conjugate (peroxidase-labeled affinity purified antibody to chicken or turkey $\mathrm{IgG}$ ) was used. The mixed antigen consisting of FP, PP and TP was prepared for screening antibodies in turkey sera but two antigens, FP and PP were combined to detect antibodies in chicken sera. Plates coated with the antigens were allowed to incubate for 1 to $16 \mathrm{hr}$ at room temperature or $4^{\circ} \mathrm{C}$. Plates were washed three times with phosphate-buffered saline (PBS) containing $0.05 \%$ Tween-20, after which $5 \mathrm{~m} l$ of $0.1 \%$ gelatin solution or $5 \%$ non-fat dry milk was added to each plate to occupy unbound sites on the plates. Plates were then washed three times with PBS, and $5 \mathrm{ml}$ of melted BiTek agar (0.6\%) in $8 \% \mathrm{NaCl}$ solution was added to each plate. The agar was allowed to harden and the pox antigen coated plates were then stored at $4^{\circ} \mathrm{C}$ for $24 \mathrm{hr}$. Serum samples collected from turkeys vaccinated with FP, PP and TP vaccines and from chickens vaccinated with FP and PP vaccines, were absorbed $(25 \mu l)$ onto round blank antibiotic disks (6 $\mathrm{mm}$ in diameter). Disks were carefully placed on the top of gel of the AGEA plates. Known positive and negative serum samples were also absorbed on disks. The plates were incubated for $4 \mathrm{hr}$ at room temperature to allow serum in the disks to diffuse through the gel and react with the poxvirus antigens. After incubation, the gels were carefully removed from the plates. The plates were washed three times with PBS, and $3 \mathrm{~m} l$ of 1:1,000 dilution of conjugate was added to each plate. The plates were incubated again at room temperature for $1 \mathrm{hr}$ and washed 3 times with PBS, after which $5 \mathrm{ml}$ of substrate $(0.4 \% 5$-Aminosalycylic acid) dissolved in $20 \mathrm{mM}$ of sodium phosphate, $\mathrm{pH} 6.8$, with $1 \%$ melted BiTek agar and $0.3 \%$ hydrogen peroxide was added to each plate. After 20 min of incubation at room temperature, reactions were read. A dark brown color indicated the presence of antibodies to FP, PP and TP while the absence of a color was considered as a negative reaction or absence of antibodies to any of the poxviruses. All serum samples screened by the AGEA were also tested by the AGPT as described $[1,8,15,20,21]$ but with slight modifications. Briefly, the AGPT was performed on microscopic slides $(3 \times 1 \mathrm{in})$ containing $3 \mathrm{ml}$ of $1 \%$ BiTek agar dissolved in $8 \% \mathrm{NaCl}$ solution. Wells were cut using a template punch and the antigen $(0.1 \mathrm{~m} l)$ was placed in the central well. The same concentrations of poxvirus antigens used for AGEA were also used in the AGPT, separately or in combination. Gels were incubated in a humidified chamber and examined after 24 and $48 \mathrm{hr}$ for precipitation lines.

The sera obtained from virus inoculated and un-inoculated chickens and turkeys were used to determine the optimal conditions for these tests. To determine the optimal conjugate concentration for each pox antigen, dilutions of $1: 200,1: 500,1: 1,000,1: 1,500$, and 1:2,000 of the peroxidase-conjugated goat anti-chicken or anti-turkey $\operatorname{IgG}$ were used. The optimal conjugate dilution to detect chicken and turkey antibodies was 1:1,000. The optimal antigen concentration to detect chicken antibodies was $0.5 \mathrm{mg} / \mathrm{ml}$ for both FP and PP antigens, but the optimal concentrations to detect turkey antibodies against FP, PP and TP were $0.1 \mathrm{mg} / \mathrm{ml}, 0.5$ $\mathrm{mg} / \mathrm{m} l$ and $1.5 \mathrm{mg} / \mathrm{ml}$, respectively, albeit $0.5 \mathrm{mg} / \mathrm{ml}$ of each antigen also detected antibodies nearly as good as the optimal concentration. Incubation of antigens for $1 \mathrm{hr}$ allowed efficient coating on the plates. To determine the optimal time for a color change after addition of the antisera, 2, 3, 4 and $24 \mathrm{hr}$ of incubation were compared. The best incubation time for a complete color change which is most visible was $4 \mathrm{hr}$. Furthermore, positive control serum (SPAFAS) raised against FPV produced a distinct, complete color change but there was no color change in areas where negative control serum (SPAFAS) was placed (Fig. 1). When individual FP,

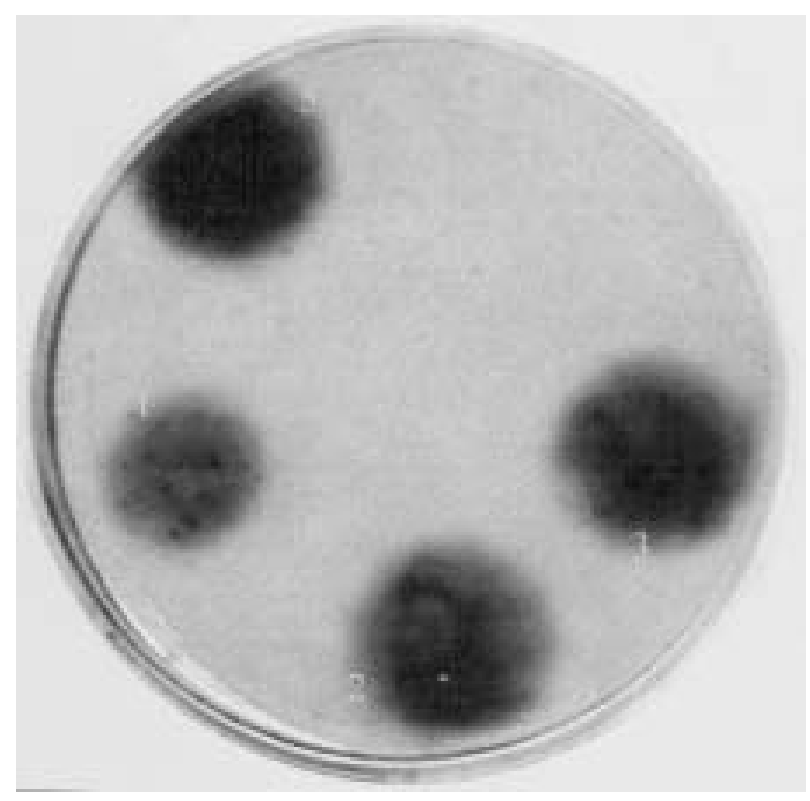

Fig. 1. Agar gel enzyme assay (AGEA) for poxvirus antibody. 1, serum from FPV inoculated chicken; 2, serum from TPV inoculated chicken; 3, serum from PPV inoculated chicken; 4, serum from CEF inoculated chicken; 5, positive FPV control serum (SPAFAS); 6, negative control serum (SPAFAS). 
Table 1. Comparison of AGPT and AGEA to detect antibodies to Pox virus in Turkey sera

\begin{tabular}{|c|c|c|c|c|c|}
\hline & \multicolumn{5}{|c|}{ AGPT } \\
\hline & \multicolumn{3}{|c|}{ ANTIGEN (FP + PP + TP) } & \multirow[t]{2}{*}{ SPECIFICITY (\%)* } & \multirow[t]{2}{*}{ SENSITIVITY (\%)** } \\
\hline & Pos & $\mathrm{Neg}$ & Total & & \\
\hline Vac & 81 & 243 & 324 & \multirow{3}{*}{100} & \multirow[t]{3}{*}{25} \\
\hline Unvac & 0 & 10 & 10 & & \\
\hline Total & 81 & 253 & 334 & & \\
\hline \multicolumn{6}{|c|}{ AGEA } \\
\hline & \multicolumn{3}{|c|}{ ANTIGEN $(\mathrm{FP}+\mathrm{PP}+\mathrm{TP})$} & SPECIFICITY (\%)* & SENSITIVITY $(\%)^{* *}$ \\
\hline & Pos & Neg & Total & \multirow{4}{*}{100} & \multirow{4}{*}{66.4} \\
\hline Vac & 215 & 109 & 324 & & \\
\hline Unvac & 0 & 10 & 10 & & \\
\hline Total & 215 & 119 & 334 & & \\
\hline
\end{tabular}

* True Negative / True Negative + False Positive.

** True Positive / True Positive + False Negative.

$\mathrm{FP}=$ Fowl pox antigen, $\mathrm{PP}=$ Pigeon pox antigen, $\mathrm{TP}=$ Turkey pox antigen.

Table 2. Comparison of AGPT and AGEA to detect antibodies to Pox virus in Chicken sera

\begin{tabular}{|c|c|c|c|c|c|}
\hline \multicolumn{6}{|c|}{ AGPT } \\
\hline & \multicolumn{3}{|c|}{ ANTIGEN (FP + PP + TP) } & SPECIFICITY (\%)* & \multirow[t]{2}{*}{ SENSITIVITY $(\%)^{* *}$} \\
\hline & Pos & $\mathrm{Neg}$ & Total & \multirow{4}{*}{100} & \\
\hline Vac & 51 & 99 & 150 & & \multirow[t]{3}{*}{34} \\
\hline Unvac & 0 & 10 & 10 & & \\
\hline Total & 51 & 109 & 160 & & \\
\hline \multicolumn{6}{|c|}{ AGEA } \\
\hline & \multicolumn{3}{|c|}{ ANTIGEN $(\mathrm{FP}+\mathrm{PP}+\mathrm{TP})$} & SPECIFICITY (\%)* & SENSITIVITY $(\%)^{* *}$ \\
\hline & Pos & $\mathrm{Neg}$ & Total & & \multirow{4}{*}{64} \\
\hline Vac & 96 & 54 & 150 & & \\
\hline Unvac & 0 & 10 & 10 & 100 & \\
\hline Total & 96 & 64 & 160 & & \\
\hline
\end{tabular}

\footnotetext{
* True Negative / True Negative + False Positive.

** True Positive / True Positive + False Negative.

$\mathrm{FP}=$ Fowl pox antigen, $\mathrm{PP}=$ Pigeon pox antigen.
}

PP or TP antigens were used in the AGPT, $6.5 \%, 3.4 \%$ and $11.7 \%$ of the turkey sera were positive for pox antibody, respectively. When the antigens were combined to form a trivalent antigen, 81 of 324 were positive by AGPT increasing the sensitivity to $25 \%$. However, out of the total 324 turkey serum samples tested by AGEA using the trivalent antigen, 215 samples were positive (Table 1), resulting in a significantly higher sensitivity of $66.4 \%(\mathrm{P}<0.001)$. When individual FP or PP antigen was used in the AGPT, $12 \%$ and $19.3 \%$ of the 150 chicken serum samples were positive for pox antibody, respectively. However, when the antigens were combined to form a bivalent antigen, 51 of the serum samples were positive increasing the sensitivity to $34 \%$, whereas using AGEA, 96 were positive (Table 2) indicating a significantly higher sensitivity of $64 \%(\mathrm{P}<0.001)$. The AGEA combines the principles of radial immunodiffusion and ELISA. The same principle have previously been reported previously $[5,7,9-11,17]$ to detect and quantify antibodies to equine rhinopneumonitis virus, pseudorabies virus, and Salmonella. In this study serum samples absorbed to the disks diffused effectively through the agar gel and formed an antigen-antibody reaction. While absorption of blank disks with serum samples have an advantage of transporting serum samples for testing, the entire AGEA procedure gives an additional advantage in that it required about $6 \mathrm{hr}$ to complete the test, whereas AGPT takes between 24 and $48 \mathrm{hr}$. Furthermore, AGEA was found to be much more sensitive when compared with AGPT. Although AGEA could be used to detect specific pox antibodies by employing individual antigens; in this study, it was used by combining two or three antigens in order to assess and screen for pox antibodies in chickens or turkeys previously infected or subjected to combined vaccines. Currently, FP, PP and TP are used to immunize commercial turkeys, and FP and PP to immunize commercial chickens against infection with poxviruses. Although these viruses 
share common specific antigens, there are still antigenic differences among them as shown by cross protection studies $[6,22]$. Such difference in antigenicity may also be determined by the AGEA using monoclonal antibodies. The idea of coating more than one antigen have also been adopted in ELISA in which three serotype specific antigens were used $[2,3]$. The sensitivity of the AGEA technique, coupled with its simplicity, makes it ideal for routine use and also for monitoring the immune level of pox vaccinated flocks, and as a satisfactory differential diagnosis of a variety of respiratory, pock lesion producing infections, such as infectious laryngeo-tracheitis (ILT) which are not macroscopically distinct from poxvirus. Furthermore, because AGEA can be quantitated $[5,7,10,17]$, it may be useful in the differentiation of the immune status of poxvirus infected versus poxvaccinated chickens or turkeys.

ACKNOWLEDGEMENT. This work was partly funded by the Southern Poultry and Egg Association.

\section{REFERENCES}

1. Baxendale, W. 1971. Vet. Rec. 88: 5-10.

2. Bosse, J.T., Johnson, R.P. and Rosendale, S. 1990. Can. J. Vet. Res. 54: 320-325.

3. Bosse, J.T., Friendship, R., Rosendale, S. and Fenwick, B.W. 1993. J. Vet. Invest. 5: 359-362.

4. Buscaglia, C., Bankowski, R.A. and Miers, L. 1985. Avian Dis. 29: $672-680$.

5. Elwing, H., Lange, S. and Nygren, H. 1980. J. Immunol. Methods 39: 247-256.

6. Gelenczei, E.F. and Lasher, H.N. 1968. Avian Dis. 12: 142-
150 .

7. Gradil, C. and Joo, H.S. 1988. Vet. Microbiol. 17: 315-322.

8. Jordan, F.T.W. and Chubb, R.C. 1962. Res. Vet. Sci. 3: 245255.

9. Johnson, E.H., Smith, B. and Reina-Guerra, M. 1984. Vet. Microbiol. 10: 71-86.

10. Joo, H.S., Molitor, T.W. and Leman, A.D. 1984. Am. J. Vet. Res. 45: 2096-2098.

11. Kim. C.J. and Nagaraja, K.V. 1991. Diagn. Microbiol. Infect. Dis. 14: 203-208.

12. Mockett, A.P.A., Southee, D.J., Tomley, F.M. and Deuter, A. 1987. Avian Pathol. 16: 493-504.

13. Nazerian, K., Dhawale, S. and Paye, W. 1989. Avian Dis. 33: 458-465.

14. Prideaux, C.T. and Boyle, D.B. 1987. Arch. Virol. 96: 185199.

15. Raj, G.D., Thangavelu, A., Govindarajan, R., Nachimuthu K. and Venugopalan, A.T. 1995. Tropical Anim. Health Prod. 27: 71-75.

16. Solomon, J.J. 1975. Tissue Culture Methods 1: 7-11.

17. Thawley, D.G., Joo, H.S., Johnson, M.E. and Solorzano, R.F. 1985. J. Am. Vet. Med. Assoc. 186: 1080-1083.

18. Tripathy, D.N., Hanson, L.E. and Myers, W.L. 1970. Avian Dis. 14: 29-38.

19. Tripathy, D.N. 1991. pp. 583-596, In: Diseases of Poultry, 9th ed. (Calnek, B.W, Barnes, J. Beard, C.W. Reid, W.M. and Yoder, H.W.eds.), Iowa State University Press, Ames, Iowa.

20. Tsubahara, K. and Kato, K. 1960. Bull. Natl. Inst. Anim. Health 41: $45-54$

21. Uppal, P.K. and Nilakantan, P.R. 1970. J. Hygiene-Cambridge 68: $349-358$.

22. Winterfield, R.W., Reed, W.M. and Thacker, H.L. 1984. Poult. Sci. 64: 2076-2080. 